\title{
The Effect of Magnesium Supplementation on Hba1c Level and Lipid Profile Among Type 2 Diabetics
}

\author{
Louay Labban* and Nasser Thallaj \\ Aljazeera Private University, Syria \\ *Corresponding Author: Louay Labban, Aljazeera Private University, Syria.
}

Received: July 31, 2019; Published: September 04, 2019

\begin{abstract}
Background: Diabetic patients usually suffer from magnesium deficiency. Increased magnesium intake may improve insulin secretion and glycemic control, and improves lipid profile. Magnesium supplementation has a clinically beneficial effect on glycemic control in type 2 diabetics. Oral magnesium supplementation has been shown to be effective in raising serum or plasma magnesium which contributes to glycemic control and improved lipid profile. The aim of this study was to evaluate the evidence of the effect of oral magnesium supplementation on glycemic control and lipid profile in patients with Type 2 diabetes.

Material and Methods: 41 individuals participated in this study 19 healthy and 22 were divided into 2 groups: group 1 (control $\mathrm{n}=19$ healthy) and group 2 (treatment $\mathrm{n}=22$ with Type 2 diabetes) were divided into 3 subgroups depending on HbA1c values. Both groups were given given $350 \mathrm{mg}$ /day oral magnesium supplementation for 8 weeks. HbA1c, TC, TG, HDL and LDL were measured in the beginning and at the end of the trial duration.

Results: 8 weeks of Magnesium supplementation increased serum Magnesium in group 2 by $22.9 \%$ and level of HbA1c was significantly lower in both groups but the reductions were greater in 3 treatment sub-groups (20.8\%). The difference in post-intervention with Magnesium supplementation in sub-groups and control group was significant $(\mathrm{P}<0.05)$. Magnesium supplementation improved lipid profile in both groups. It decreased TC (16\%), TG (15.8\%), LDL-C (20.9\%) and increased HDL-C (20.6\%) and subsequently improved LDL-C/HDL-C ratio. The difference between control and treatment groups of all parameters was statistically significant.

Conclusion: Oral Magnesium supplementation for 8 weeks shown to be effective in reducing HbA1c, TC, TG, LDL levels and raising HDL level in patients with Type 2 diabetes and can be considered as prevention and treatment of DM type 2 and its complications. The daily diet should include a dietary source of magnesium or a supplement.
\end{abstract}

Keywords: Magnesium; HbA1c; LDL-C; TG; TC; HDL-C

\section{Introduction}

Magnesium is an abundant macro-element in the human body and plays an important role in many biological processes. Normal magnesium values are between $1.8-2.3 \mathrm{mg} / \mathrm{dL}$ and its deficiency is defined as a drop in its normal serum concentrations below 1.7 $\mathrm{mg} / \mathrm{dL}$ [1]. Magnesium deficiency can cause many chronic diseases such as Diabetes, hypertension, and cardiovascular diseases. The most common health issue which associated to Magnesium deficiency is Diabetes Mellitus in addition to many other problems [2]. It's estimated that one-third of type 2 diabetics have low levels of Magnesium which is caused mainly by enhanced renal excretion of Magnesium [3]. There are several causes for low Magnesium levels in diabetics including diets low in Magnesium, osmotic diuresis that leads to high renal excretion of Magnesium, These deficits have been also linked to other complications such as development of atherosclerosis, $[4,5]$ and in patients with coronary atherosclerosis, a Magnesium deficit has been related to an atherogenic lipid profile [6].
Many studies have shown that Magnesium supplementation improves insulin sensitivity [7]. Dietary supplementation with Magnesium in addition to classical therapies for diabetes may help in prevention or delaying of diabetic complications [8]. Oral Magnesium supplementation improves insulin sensitivity and metabolic control in type 2 diabetics with lower serum Magnesium levels. It has also been shown that it has beneficial effect on lipid profile of diabetic patients $[9,10]$.

The aim of this study was to measure the levels of Magnesium in serum of type 2 diabetics and to find out its relationship to Hba1c and lipid profile. The other aim was to evaluate the effect of oral Magnesium supplementation on the improvement in Hba1c values and in lipid profile parameters.

\section{Material and Methods}

The study was carried out between March and May 2018 on 41 type 2 diabetic participants ( 21 males and 20 females). The inclu- 
sion criteria for enrolled participants were: previous diagnosis with Type 2 diabetes, age between 28 and 58 years, males and females. Whereas the exclusion criteria were: Patients who have renal disease diagnosed by serum urea and creatinine test, diuretics usage in the last 2 weeks and patients with persistent diarrhea and vomiting.

The participants were divided into 2 groups:

- Group I: Control group consisted of 19 participants which had 9 males and 10 females. The participants of this group were healthy and their HbA1c and lipid profile values were within the normal range.

- Group II: Study group consisted of 22 Participants. This group had 12 males and 10 females.

The second group or the study group was divided into 3 subgroups according to the $\mathrm{HbA} 1 \mathrm{c}$ values as follows:

- Subgroup 1: HbA1c 6 - 7 (7 participants)

- Subgroup 2: HbA1c 7-8 (7 participants)

- $\quad$ Subgroup 3: HbA1c > 8 (8 participants).

In order to measure Magnesium serum value and lipid profile, COBAS Intergra 400 plus (Roche, Germany) was used during this study.

Prior to enrollment in this study, a signed consent was obtained from all participants. Blood samples were drawn twice from all participants at the beginning and at the end of this study in order to measure serum Magnesium, HbA1c, and lipid profile. A profes- sional nurse drew blood samples under supervision of lab supervisor. 300 mg Magnesium of oral Magnesium oxide supplementation were given daily for 8 weeks (Magnesium oxide was manufactured by Douglas lab (USA) 250 tablets per container.

All data were statistically analyzed using SPSS 15.0 for windows (SPSS Inc., Chicago, IL). Age and serum Magnesium, LDL-C, HDL-C, $\mathrm{TG}, \mathrm{TC}$ of participants were expressed as the mean $\pm \mathrm{SD}$ and median (range), and improvements in Magnesium levels, Hba1c levels and lipid profile were expressed as percentage and a P value of $<0.05$ was considered significant.

\section{Results}

The results of this study have shown that the mean age of the participants was $43.18 \pm 15.29$ years and the mean serum Magnesium level among all participants before supplementation was $1.61 \pm 0.46 \mathrm{mg} / \mathrm{dL}$. According to the cut-off level of normal serum Magnesium $(1.7 \mathrm{mg} / \mathrm{dL})$ the values indicate that most participants $(88.3 \%)$ were magnesium deficient. Before supplementation, they were more deficient females than males $(89.3 \%$ and $87.2 \%$ respectively).

Serum Magnesium level has gone up after supplementation among all participants from $1.61 \pm 0.46 \mathrm{mg} / \mathrm{dL}$ to $1.64 \pm 0.51 \mathrm{mg} /$ $\mathrm{dL}$. The highest increase were among females (11.8\%) comparing with males (4.6\%). When comparing groups, the study group had the highest increase in serum Magnesium level comparing with the control group. The increase was $22.5 \%$ and $1.9 \%$ respectively and the difference was statistically significant $(\mathrm{P}<0.05)$. These results are shown in Table 1 and Table 2.

\begin{tabular}{|l|c|c|c|c|c|c|}
\hline & Males & Improvement & Females & Improvement & All participants & Improvement \\
\hline Before & $1.53 \pm 0.41$ & \multirow{2}{*}{$4.6 \%$} & $1.36 \pm 0.33$ & \multirow{2}{*}{$19.1 \%$} & $1.61 \pm 0.46$ & \multirow{2}{*}{$8 \%$} \\
\cline { 1 - 1 } After & $1.59 \pm 0.43^{\mathrm{c}}$ & & $1.62 \pm 0.41^{\mathrm{a}}$ & & $1.74 \pm 0.51^{\mathrm{b}}$ & \\
\hline
\end{tabular}

Table 1: Serum Magnesium levels differences between genders before and after supplementation.

* Different letter denotes significant difference $(\mathrm{P}<0.05)$.

\begin{tabular}{|l|l|l|l|l|}
\hline & Group1 & \% improvement & Group 2 & \% improvement \\
\hline Before & $1.61 \pm 0.53$ & $1.9 \%$ & $1.38 \pm 0.49$ & $22.9 \%$ \\
\cline { 1 - 1 } After & $1.64 \pm 0.61^{\mathrm{b}}$ & & $1.79 \pm 0.56^{\mathrm{a}}$ & \\
\hline
\end{tabular}

Table 2: The improvement in serum Magnesium level in groups after supplementation.

* Different letter denotes significant difference $(\mathrm{P}<0.05)$.

The data demonstrated in Table 3, has shown strong correlations between serum Magnesium and HbA1c values within groups (control and study groups) alike. Supplementation of Magnesium resulted in 8.8\% improvement in HbA1c values in group I (Healthy group) whereas the highest improvement (20.8\% reduction) was seen among participants who had Hba1c higher than 8 followed by those with HbA1c values 7-8 and the slightest decrease was in sub-group with HbA1c values 6-7. The difference was statistically significant between group I and II $(\mathrm{P}<0.05)$.

Table 4 shows that there was statistically significant difference in TG between group I and group II $(\mathrm{P}<0.05)$. The reduction in TG value in group I was $6.9 \%$. This reduction was higher in group II after Magnesium supplementation. 


\begin{tabular}{|c|c|c|c|c|c|c|c|c|}
\hline \multirow{2}{*}{ HbA1c Values } & \multirow{2}{*}{\multicolumn{2}{|c|}{$\begin{array}{c}\text { Group I } \\
\text { Healthy }\end{array}$}} & \multicolumn{6}{|c|}{ Group II } \\
\hline & & & \multicolumn{2}{|c|}{ HbA1c 6- 7} & \multicolumn{2}{|c|}{ HbA1c 7- 8} & \multicolumn{2}{|c|}{ HbA1c $>8$} \\
\hline Before & $5.7 \pm 1.23$ & $8.8 \%$ & $6.9 \pm 1.54$ & $10.1 \%$ & $7.8 \pm 1.67$ & $17.9 \%$ & $9.6 \pm 1.69$ & $20.8 \%$ \\
\hline After & $5.3 \pm 1.11^{d}$ & & $6.2 \pm 1.43^{c}$ & & $6.4 \pm 1.53^{b}$ & & $7.6 \pm 1.45^{\mathrm{a}}$ & \\
\hline
\end{tabular}

Table 3: The effect of Magnesium supplementation on HbA1c values.

* Different letter denotes significant difference $(P<0.05)$.

\begin{tabular}{|l|c|c|c|c|}
\hline \multicolumn{2}{|c|}{ Group } & Supplementation & Mean serum TG & \% Improvement \\
\hline \multirow{3}{*}{ Group I } & Healthy & Before & $175.1 \pm 23.4$ & \multirow{2}{*}{6.9} \\
\cline { 3 - 4 } & & After & $163 \pm 21.2^{\mathrm{b}}$ & \\
\hline \multirow{3}{*}{ Group II } & HbA1c 6-7 & Before & $171.8 \pm 23.2$ & \multirow{2}{*}{4.8} \\
\cline { 3 - 4 } & & After & $163.6 \pm 20.9^{\mathrm{b}}$ & \multirow{2}{*}{11.9} \\
\cline { 3 - 4 } & HbA1c 7-8 & Before & $174.4 \pm 27.1$ & \multirow{2}{*}{1} \\
\cline { 3 - 4 } & & After & $153.6 \pm 25.3^{\mathrm{a}}$ & \multirow{2}{*}{15.8} \\
\cline { 3 - 4 } & \multirow{2}{*}{ HbA1c >8 } & Before & $179.2 \pm 26.1$ & \\
\cline { 3 - 4 } & & After & $150.8 \pm 19.2^{\mathrm{a}}$ & \\
\hline
\end{tabular}

Table 4: Mean Serum TG of the groups before and after supplementation.

* Different letter denotes significant difference $(P<0.05)$.

Supplementation of Magnesium resulted in improvement in low density lipoprotein (LDL-C) value in both groups. But this improvement was greater in group II and especially in those with mean serum HbA1c more than 8 . The reduction was $20.9 \%$. The difference was statistically significant $(\mathrm{P}<0.05)$. The results are shown in table 5.
With regard to total cholesterol, the data obtained from this study showed that Magnesium supplementation improved total cholesterol in group II only. The reduction was greater in subgroup 3 (Hba1c $>8$ ) and the reduction was $16 \%$ comparing to $-0.7 \%$ in group I. The difference was statistically significant $(\mathrm{P}<0.05)$ as seen in table 6.

\begin{tabular}{|c|c|c|c|c|}
\hline \multicolumn{2}{|c|}{ Group } & Supplementation & Mean Serum LDL-C & $\%$ Improvement \\
\hline \multirow[t]{2}{*}{ Group I } & \multirow{2}{*}{ Healthy } & Before & $141.7 \pm 18.3$ & \multirow{2}{*}{6.9} \\
\hline & & After & $131.9 \pm 18.1^{\mathrm{d}}$ & \\
\hline \multirow[t]{6}{*}{ Group II } & \multirow{2}{*}{ HbA1c 6-7 } & Before & $139.8 \pm 17.3$ & \multirow{2}{*}{6.2} \\
\hline & & After & $131.2 \pm 15.8^{\mathrm{c}}$ & \\
\hline & \multirow{2}{*}{ HbA1c 7-8 } & Before & $133.7 \pm 15.6$ & \multirow{2}{*}{4.7} \\
\hline & & After & $127.4 \pm 16.2^{b}$ & \\
\hline & \multirow{2}{*}{$\mathrm{HbA} 1 \mathrm{c}>8$} & Before & $151.6 \pm 14.1$ & \multirow{2}{*}{20.9} \\
\hline & & After & $119.9 \pm 11.3^{\mathrm{a}}$ & \\
\hline
\end{tabular}

Table 5: Serum LDL-C improvements of the groups.

* Different letter denotes significant difference $(P<0.05)$.

\begin{tabular}{|c|c|c|c|c|}
\hline \multicolumn{2}{|c|}{ Group } & Supplementation & Mean Serum TC & \% Improvement \\
\hline \multirow[t]{2}{*}{ Group I } & \multirow{2}{*}{ Healthy } & Before & $215.6 \pm 19.3$ & \multirow{2}{*}{-0.7} \\
\hline & & After & $217.2 \pm 18.6^{d}$ & \\
\hline \multirow[t]{6}{*}{ Group II } & \multirow{2}{*}{ HbA1c 6-7 } & Before & $223.4 \pm 18.3$ & \multirow{2}{*}{5.6} \\
\hline & & After & $210.8 \pm 17.4^{c}$ & \\
\hline & \multirow{2}{*}{ HbA1c 7-8 } & Before & $231.5 \pm 16.2$ & \multirow{2}{*}{11.5} \\
\hline & & After & $204.8 \pm 16.1^{b}$ & \\
\hline & \multirow{2}{*}{$\mathrm{HbA} 1 \mathrm{c}>8$} & Before & $232.6 \pm 16.9$ & \multirow{2}{*}{16} \\
\hline & & After & $195.4 \pm 14.8^{\mathrm{a}}$ & \\
\hline
\end{tabular}

Table 6: Serum TC improvements of the groups.

* Different letter denotes significant difference $(P<0.05)$. 
Table 7 shows that Magnesium supplementation is positively correlated with high density lipoprotein (HDL-C). The increase was in both groups (I and II) but the highest increase was in group II and more specifically in those with HbA1c value more than 8. The difference was statistically significant $(\mathrm{P}<0.05)$.
As Magnesium supplementation has improved mean LDL-C, HDL-C and total cholesterol (TC) values, these improvements have been reflected in LDL-C/HDL-C ratio as shown in table 8. The best ratio was in group II.

\begin{tabular}{|c|c|c|c|c|}
\hline \multicolumn{2}{|c|}{ Group } & Supplementation & Mean Serum HDL-C & \% Improvement \\
\hline \multirow[t]{2}{*}{ Group I } & \multirow{2}{*}{ Healthy } & Before & $40.8 \pm 2.8$ & \multirow{2}{*}{8.5} \\
\hline & & After & $44.6 \pm 2.1^{\mathrm{c}}$ & \\
\hline \multirow[t]{6}{*}{ Group II } & \multirow{2}{*}{ HbA1c 6-7 } & Before & $40.1 \pm 2.4$ & \multirow{2}{*}{4.3} \\
\hline & & After & $41.9 \pm 2.4^{\mathrm{d}}$ & \\
\hline & \multirow{2}{*}{ HbA1c 7-8 } & Before & $40.1 \pm 1.9$ & \multirow{2}{*}{14.3} \\
\hline & & After & $46.8 \pm 2.2^{b}$ & \\
\hline & \multirow{2}{*}{$\mathrm{HbA} 1 \mathrm{c}>8$} & Before & $40.2 \pm 2.7$ & \multirow{2}{*}{20.6} \\
\hline & & After & $50.6 \pm 2.9^{a}$ & \\
\hline
\end{tabular}

Table 7: Serum HDL-C improvements of groups.

* Different letter denotes significant difference $(P<0.05)$.

\begin{tabular}{|l|c|c|c|c|}
\hline \multicolumn{2}{|c|}{ Group } & Supplementation & LDL-C/HDL-C Ratio & \% Improvement \\
\hline \multirow{3}{*}{ Group I } & \multirow{2}{*}{ Healthy } & Before & 3.47 & \multirow{2}{*}{13.5} \\
\cline { 3 - 4 } & \multirow{3}{*}{ Group II } & After & $3^{\mathrm{c}}$ & \multirow{2}{*}{14.1} \\
\cline { 3 - 4 } & \multirow{3}{*}{ HbA1c 6-7 } & Before & 3.48 & \multirow{2}{*}{18.3} \\
\cline { 2 - 4 } & \multirow{2}{*}{ HbA1c 7-8 } & After & $3.05^{\mathrm{d}}$ & \multirow{2}{*}{24.1} \\
\cline { 2 - 4 } & \multirow{2}{*}{ HbA1c >8 } & Before & $2.72^{\mathrm{b}}$ \\
\cline { 2 - 4 } & & After & 3.11 & $2.36^{\mathrm{a}}$ \\
\hline
\end{tabular}

Table 8: LDL-C/HDL-C ratio of the groups.

\section{Discussion}

To the best of our knowledge, this is a pioneer study to link Magnesium supplementation to improvement of glucose level and lipid profile parameters in order to reduce diabetic complications .As many studies have demonstrated that Magnesium is an important cation which plays a significant role in glucose and insulin metabolism, mainly through its impact on tyrosine kinase enzyme. Magnesium may also directly affect glucose transporter protein activity 4 and regulate glucose translocation into the cell. The data obtained from this study that Magnesium supplementation positively correlated with reduction in HbA1c values. This finding is similar to study carried out by Galli-Tsinopoulou., et al. [11]. Ramadass., et al. [12] Sinha and Sen [13] showed similar results in adult patients with type 2 diabetes.

There was statistically significant difference in serum lipids level after Magnesium supplementation in the study group (group II). Magnesium supplementation reduced mean serum TG, TC, and LDL-C levels but increases HDL-C levels. The best results were in group II and more specifically in those with $\mathrm{HbA1c}$ level higher than 8. These outcomes are in concordance with [13] and Mishra., et al. [14]. Guerrero-Romero and Rodríguez-Morán [8] showed that in type 2 diabetics, low magnesium is linked with low levels of HDL.
The data obtained from this study showed that there was statistically significant difference in serum Magnesium level before and after Magnesium supplementation being higher after Magnesium supplementation with mean \pm SD of Magnesium was $1.64 \pm 0.61$ Magnesium/dL before Magnesium supplementation versus $1.79 \pm$ 0.56 Magnesium/dL after Magnesium supplementation $(\mathrm{P}<0.05)$. This is in agreement with Rodriguez-Moran and Guerrero- Romero [8] in type 2 diabetic adults revealed that there is a significant increase in serum Magnesium after a period of oral Magnesium supplementation who revealed increase in serum Magnesium level with Magnesium supplementation (500 Magnesium twice daily of Magnesium Oxide) for 24 weeks in adult patients with type 1 diabetes.

Our study showed that there was a significant difference between $\mathrm{HbA1c}$ before and after Magnesium supplementation as mean value of $\mathrm{HbA1} \mathrm{c}$ after supplementation. The greater improvement in HbA1c values was in group II in those with HbA1c value higher than 8. This indicates that oral Magnesium supplementation may play a good role on glycemic control diabetes [14]. This result is concordant with Rodriguez-Moran and Guerrero-Romero [8] who showed that Magnesium chloride supplementation resulted in significant reduction of $\mathrm{HbA1c}$. 
Lopez-Ridaura., et al. [15] also showed that Magnesium supplementation decreases the risk of type 2 diabetes in adult population.

Meta-analysis study was done by Simental-Mendía., et al. [16] to observe the effect of oral Magnesium supplementation on insulin sensitivity and glucose control in both diabetic and non-diabetic individuals. In this study, there was a significant difference in lipid parameters after oral Magnesium supplementation. This is in agreement with Djurhuus., et al. [17] and [18] observed decreased atherogenic lipid fractions (total cholesterol, LDL, and Apo lipoprotein B), with Magnesium supplementation. Also Lal., et al. [19] observed a significant fall in serum total cholesterol, LDL, and triglycerides and a rise in HDL 4 to 8 weeks after Magnesium supplementation which consistent with our study also Solati., et al. [20-23] showed a significant reduction in LDL in Magnesium supplemented diabetic patients.

\section{Conclusion}

This study have shown that Magnesium deficiency is widely spread in type 2 diabetics, and it is supplementation is correlated with glycemic control and lipid profile. The study has also concluded that correction of low Magnesium in type 2 diabetics with oral Magnesium supplements is associated with optimization of glycemic control and improvement in lipid profile. Therefore, Magnesium supplementation can be used as prevention of type 2 diabetes or as a tool of glycemic control and improvement of lipid profile parameters among type 2 diabetics and as a prevention of diabetic complications such as cardiovascular diseases.

\section{Recommendations}

As it has shown that Magnesium plays an important role in improving HbA1c level in general and glycemic control in particular in type 2 diabetics and also has an important role in reducing LDLC, TC, TG levels and increasing HDL-C levels. It's recommended that our meals should contain adequate amount of magnesium from its natural resource. If this difficult, supplements remain another option. The RDA for Magnesium is 310-420 mg for adults depending on age and gender. The main Magnesium sources are Whole Wheat, spinach, quinoa, almonds, cashews, peanuts, dark Chocolate, black Beans, edamame and avocado.

\section{Bibliography}

1. Fang X., et al. "Dietary magnesium intake and the risk of cardiovascular disease, type 2 diabetes, and all-cause mortality: a dose response meta-analysis of prospective cohort studies". BMC Medicine 14 (2016): 210.

2. Rude RK. "Magnesium deficiency and diabetes mellitus. Causes and effects". Postgraduate Medical Journal 92.5 (1992): 217-224.

3. Dasgupta A., et al. "Hypomagnesemia in type 2 diabetes mellitus". Indian Journal of Endocrinology and Metabolism 16.6 (2012):1000-1003.
4. Johansson G., et al. "Evidence for a disturbed magnesium metabolism in diabetes mellitus". Magnes Bull 2 (1981): 178-180.

5. Tapiero $\mathrm{H}$ and Tew KD. "Trace elements in human physiology and pathology: zinc and metallothioneins". Biomedicine and Pharmacotherapy 57.9 (2003): 399-411.

6. Deckert T., et al. "Albuminuria reflects widespread vascular damage. The Steno hypothesis". Diabetologia 32.4 (1989): 219-226.

7. Gropper SS., et al. "Magnesium. Advanced nutrition and human metabolism". 4th edition. Belmont, CA: Thomson and Wadsworth (2005).

8. Rodríguez-Morán M and Guerrero-Romero F. "Oral magnesium supplementation improves insulin sensitivity and metabolic control in type 2 diabetic subjects: a randomized doubleblind controlled trial”. Diabetes Care 26.4 (2003):1147-1152.

9. Agrawal P., et al. "Association of macrovascular complications of type 2 diabetes mellitus with serum magnesium levels". Diabetes and Metabolic Syndrome 5.1 (2011): 41-44.

10. Wegner M., et al. "The relationship between concentrations of magnesium and oxidized low density lipoprotein and the activity of platelet activating factor acetylhydrolase in the serum of patients with type 1 diabetes". Magnesium Research 23.2 (2010): 97-104.

11. Galli-Tsinopoulou A., et al. "Association between magnesium concentration and $\mathrm{HbA1c}$ in children and adolescents with type 1 diabetes mellitus". Journal of Diabetes 6.4 (2014): 369 377.

12. Ramadass S., et al. "Serum magnesium level as an indicator of status of diabetes mellitus type 2". Diabetology and Metabolic Syndrome 9.1 (2015): 42-45.

13. Sinha S and Sen S. "Status of zinc and magnesium levels in type 2 diabetes mellitus and its relationship with glycemic status". International Journal of Diabetes in Developing Countries 34.4 (2014): 220-223.

14. Srinivasan AR., et al. "Status of serum magnesium in type 2 diabetes mellitus with particular reference to serum triacylglycerol levels". Diabetes and Metabolic Syndrome 6.4 (2012): 187-189.

15. Rodríguez-Morán M and Guerrero-Romero F. “Oral magnesium supplementation improves insulin sensitivity and metabolic control in type 2 diabetic subjects: a randomized double-blind controlled trial". Diabetes Care 26 (2003): 1147-1152.

16. Guerrero-Romero F and Rodríguez-Morán M. "Hypomagnesemia is linked to low serum HDL-cholesterol irrespective of serum glucose values". Journal of Diabetes and its Complications 14.5 (2000): 272-276 
17. Lopez-RidauraR., et al. "Magnesiumintake and risk of type 2 diabetes in men and women". Diabetes Care 27.1 (2004): 134140 .

18. Simental-Mendía LE., et al. "A systematic review and metaanalysis of randomized controlled trials on the effects of magnesium supplementation on insulin sensitivity and glucose control". Pharmacological Research 111 (2016): 272-282.

19. Djurhuus MS., et al. "Magnesium reduces insulin-stimulated glucose uptake and serum lipid concentrations in type 1 diabetes". Metabolism 50.12 (2001):1409-1417.

20. Sjogren A., et al. "Oral administration of magnesium hydroxide to subjects with insulin-dependent diabetes mellitus: effects on magnesium and potassium levels and on insulin requirements". Magnesium 7.3 (1988): 117-122.

21. Lal J., et al. "Effect of oral magnesium supplementation on the lipid profile and blood glucose of patients with type 2 diabetes mellitus". Journal of the Association of Physicians of India 51 (2003): 37-42.

22. Mishra S., et al. "Serum magnesium and dyslipidemia in type- 2 diabetes mellitus". Biomedical Research 23.2 (2012): 295-300.

23. Solati M., et al. "Oral magnesium supplementation in type II diabetic patients". Medical Journal of The Islamic Republic of Iran 28 (2014): 67.

Volume 3 Issue 10 October 2019

(C) All rights are reserved by Louay Labban and Nasser

Thallaj. 